\title{
Control of Underactuated Planar Hexapedal Pronking Through a Dynamically Embedded SLIP Monopod
}

\author{
M. Mert Ankaralı, Uluc Saranlı and Afsar Saranlı
}

\begin{abstract}
Pronking (aka. stotting) is a gait in which all legs are used in synchrony, resulting in long flight phases and large jumping heights that may potentially be useful for mobile robots on rough terrain. Robotic instantiations of this gait suffer from severe pitch instability either due to underactuation, or the lack of sufficient feedback. Nevertheless, the dynamic nature of this gait suggests that the Spring-Loaded Inverted Pendulum Model (SLIP), a very successful predictive model for both natural and robotic runners, would be a good basis for more robust and maneuverable robotic pronking. In this paper, we describe how "template-based control", a controller structure based on the embedding of a simple dynamical "template" within a more complex "anchor" system, can be used to achieve stable and controllable pronking for a planar, underactuated hexapod model. In this context, high-level control of the gait is regulated through speed and height commands to the SLIP template, while the embedding controller based on approximate inversedynamics and carefully designed passive dynamics ensures the stability of the remaining degrees of freedom. We show through extensive simulation experiments that unlike existing open-loop alternatives, the resulting control structure provides stability, explicit maneuverability and significant robustness against sensor and actuator noise.
\end{abstract}

\section{INTRODUCTION}

\section{A. Motivation and Background}

Pronking is a gait often adopted by legged animals to signal their strength to potential predators $[8,12]$, with all legs used in synchrony and a substantial flight phase induced (see Fig. 1). For robotic platforms, the large jumping heights associated with this gait may be useful for locomotion on cluttered natural environments and may even increase efficiency by decreasing damping losses. Moreover, the lateral symmetry of the gait admits the use of simple planar models and provides a rich domain for studying feedback control of dynamic legged locomotion. Such a simplification also allows the analysis of structurally similar gaits such as the trot and the pace [4].

Existing pronking controllers often use fully actuated leg designs. However, despite associated mobility and control advantages, the resulting electromechanical complexity significantly impairs performance for autonomous outdoor operation [18]. In contrast, robots with carefully designed passive compliant dynamics showed that a large pallet of behaviors are still possible with very few actuators [2, 20, 26]. Consequently, our emphasis in this paper is on how

M. M. Ankaralı is with the Dept. of Elec. \& Electronics Eng., Middle East Tech. Univ., 06531 Ankara, Turkey ankarali deee.metu.edu.tr

U. Saranlı is with the Dept. of Comp. Eng., Bilkent University, 06800 Ankara, Turkey saranlidcs.bilkent.edu.tr

A. Saranlı is with the Dept. of Elec. \& Electronics Eng., Middle East Tech. Univ., 06531 Ankara, Turkey afsars@metu.edu.tr

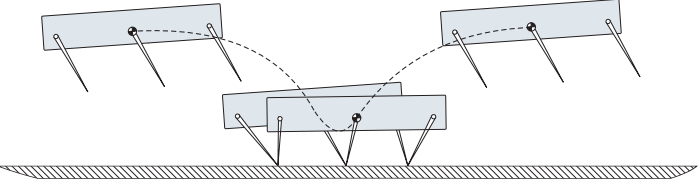

Fig. 1. Snapshot of a pronking stride for a planar hexapod.

robust and maneuverable pronking can be obtained with underactuated legged robots, such as the RHex hexapod [23].

Regardless of available actuation, stable and maneuverable control of pronking is a difficult problem. Open-loop controllers suffer from severe pitch instability and even the addition of low-bandwidth sensory components does not yield sufficient robustness for autonomous operation [17]. In fact, pronking dynamics under simple energy-based feedback and largely open-loop leg control was shown to be inherently unstable for certain ranges of body inertia and locomotion heights [5]. Even though it is possible to obtain stable pronking and other similar gaits by careful co-optimization of morphological and control parameters [9], maneuverability still remains limited, motivating the present study.

In this paper, we propose a method for highly maneuverable control of the pronk through a careful "anchoring" [13] of the Spring-Loaded Inverted Pendulum (SLIP) "template" into a complex planar hexapod model. Our approach closely parallels the ideas introduced in [22] but includes specific extensions for the pronking behavior as well as a careful characterization of how robust the resulting controller is against noise. A similar application for a 3DOF asymmetric hopper was described in [19]. In the last two decades, the SLIP model, illustrated in Fig. 2, has been established as a successful descriptive model for dynamic running behaviors $[1,7,27]$. Its morphology has been successfully used for several running monopods [15,20,26,28], while principles behind its descriptive success have been used to design robots with more complex morphologies $[3,20]$. In the present paper, we extend formal results on the analysis and control of the SLIP model and use them for controlling pronking.

\section{B. Methodology and Contributions}

Our method is based on decomposing system degrees of freedom into two components: A dynamical template, handling degrees of freedom relevant to the description and control of the high level task, and the anchor, encompassing the remaining degrees of freedom. Having been successfully applied to the control of alternating tripod gaits, this idea is not only appealing from an engineering perspective [24], but is also supported by experimental data demonstrating the ability of simple models to accurately capture biomechanical 


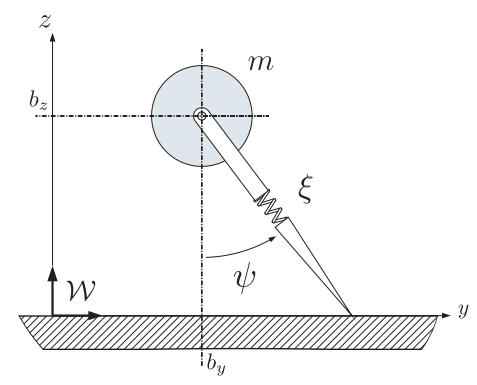

Fig. 2. The template SLIP model

data [13]. A similar decomposition was successfully applied to the control of bipedal walking [16] and running [10] through the use of zero-dynamics and optimization methods.

The suitability of the SLIP model as a template for locomotion tasks is established through both its consistent and undeniable identification in biological systems $[6,7,11]$, as well as due to the presence of numerous control strategies that have been proposed for this system [14,20,25]. These controllers are capable of very accurate realization and tracking of high level commands in the form of desired forward velocity and hopping height, presenting a very convenient and maneuverable interface to pronking.

Our primary contribution in this paper is the application of the template-based control idea to pronking and a careful simulation study to document stability and robustness properties of the resulting controller under various noise conditions.

\section{Dynamics And Control of SLIP}

\section{A. System Model}

The SLIP model consists of a point mass $m$ and a freely rotating massless leg, endowed with a passive, linear spring-damper pair of compliance $k_{s}$ and viscous damping $d_{s}$. Throughout locomotion, the model alternates between stance and flight phases, which are further divided into the compression, decompression and ascent, descent subphases, respectively. In flight, the body is assumed to be a projectile acted upon by gravity, whereas in stance the toe is assumed to be fixed on the ground with the body mass feeling radial forces induced by the leg. Table I details the notation we use for the SLIP model.

TABLE I

\section{SLIP MODEL NOTATION}

\begin{tabular}{|c|l|}
\hline \multicolumn{2}{|c|}{ SLIP States and Parameters } \\
\hline$\xi, \psi, \dot{\xi}, \dot{\psi}$ & Leg length, leg angle and their derivatives \\
$k_{s}, \xi_{0}$ & leg spring stiffness and rest length \\
$\xi_{t d}, \psi_{t d}, t_{t d}$ & Leg length, angle and time at touchdown \\
$\xi_{l o}, \psi_{l o}, t_{l o}$ & Leg length, angle and time at liftoff \\
$b_{z_{a}}, b_{\dot{y}_{a}}$ & Apex height and apex horizontal velocity \\
\hline
\end{tabular}

Controlling SLIP locomotion can be achieved with a variety of control inputs [27]. In this paper, we use the leg touchdown angle $\psi_{t d}$ and leg lengths at touchdown $\xi_{t d}$ and liftoff $\xi_{l o}$. This set of control inputs not only makes stance dynamics fully passive [28], but also admits their embedding using RHex's underactuated leg morphology [24] through explicit placement of a "virtual toe" at touchdown.

\section{B. Deadbeat Stride Control}

In this section, we briefly describe the analytical stance map for the SLIP model introduced in [14], and propose an associated single-step deadbeat controller for high-level control of locomotion.

Two of the control inputs, the touchdown and liftoff leg lengths can be easily computed using the desired energy difference $\Delta E$ between successive apex states. In particular, if $\Delta E>0$, the leg is precompressed during flight to yield

$$
\begin{aligned}
& \xi_{t d}=\xi_{0}-\sqrt{2 \Delta E / k_{s}}, \\
& \xi_{l o}=\xi_{0} .
\end{aligned}
$$

Similarly, if $\Delta E<0, \xi_{l o}$ is shortened accordingly while $\xi_{t d}$ is kept equal to the rest length. It is important to note that these derivations pertain to the ideal SLIP model, for which the stance phase is lossless. In our embedding of this ideal SLIP in the hexapod model, we will introduce further energy based corrections to explicitly account for the effects of damping and embedding inaccuracies.

Computation of the touchdown leg angle requires the derivation of an accurate stance map for the SLIP model. As described in [14], the effect of gravity on the lossless stance dynamics can be linearized such that the angular momentum and total mechanical energy become constants of motion. Based on these assumptions, approximate expressions for stance trajectories can be written as

$$
\begin{aligned}
\xi(t)= & \xi_{0}\left(1+a+b \sin \left(\hat{\omega}_{0} t\right)\right) \\
\psi(t)= & \psi_{t d}+\omega(1-2 a)\left(t-t_{t d}\right) \\
& +\frac{2 b \omega}{\hat{\omega}_{0}}\left[\cos \left(\hat{\omega}_{0} t\right)-\cos \left(\hat{\omega}_{0} t_{t d}\right)\right],
\end{aligned}
$$

where $a, b, \hat{\omega}_{0}$ and $\omega$ are constants that depend on system parameters and touchdown states as detailed in [14]. These solutions are formulated with respect to an unknown time origin but (3) can be used together with the leg length control inputs to identify associated time instants as

$$
\begin{aligned}
& t_{t d}=\left(\pi-\arcsin \left(\left(\xi_{t d} / \xi_{0}-1-a\right) / b\right)\right) / \hat{\omega}_{0}, \\
& t_{l o}=\left(2 \pi+\arcsin \left(\left(\xi_{l o} / \xi_{0}-1-a\right) / b\right)\right) / \hat{\omega}_{0},
\end{aligned}
$$

extending the derivations of [14] where transition leg lengths were always assumed to be equal to the leg rest length. Once these times are computed, an analytical approximation for the entire apex return map $\hat{f}_{a}\left(\psi_{t d}\right)$ can be written.

In all of our simulations, we have observed that this onedimensional return map is monotonic in $\psi_{t d}$ and is hence invertible. Unfortunately, this inverse cannot be obtained analytically. Nevertheless, the only remaining input is the touchdown leg angle $\psi_{t d}$ for which a numerical solution is trivial and yields the desired forward velocity $b^{*} \dot{y}_{a}$. Formally, we solve the minimization problem

$$
\psi_{t d}=\underset{\frac{-\pi}{2}<\psi<\frac{-\pi}{2}}{\operatorname{argmin}}\left(b^{*} \dot{y}_{a}-\left(\pi_{b_{\dot{y}_{a}}} \circ \hat{f}_{a}\right)(\psi)\right)^{2},
$$

where $\pi_{\dot{b}_{y a}}$ operator retrieves the forward velocity component of the return map. This results in an effective, step-based deadbeat controller for the SLIP model. 


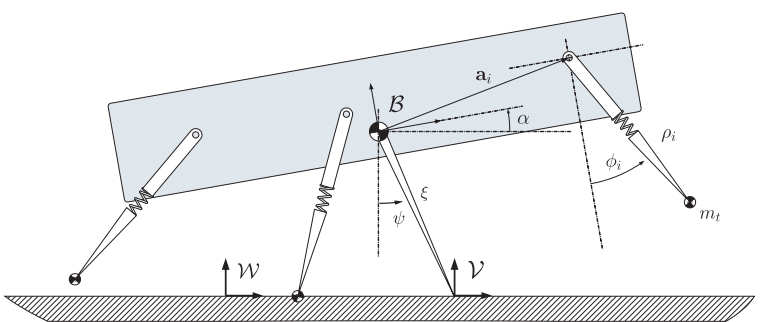

Fig. 3. Slimpod: A planar dynamic model for hexapedal pronking

\section{The Planar HeXapod Model}

Due to the dominant sagittal symmetry of pronking, we adopt a planar approximation (the Slimpod model [22]) both in our derivations and our simulations. Our hypothesis is that planarity can be ensured in practice through laterally differential leg torque adjustments. We now describe this model, its dynamics and associated simplifications.

\section{A. System Model}

The Slimpod model, illustrated in Fig. 3, consists of a rigid body with three compliant legs, each of which represents a sagittally symmetric pair of legs. We also define a "virtual leg" extending from the body center of mass (COM) to the ground which we will use for our embedding controller. We define a fixed inertial frame $\mathcal{W}$, a body reference frame $\mathcal{B}$ located at the COM and finally a virtual toe frame $\mathcal{V}$, whose orientation is the same as $\mathcal{W}$, located at the virtual toe. Legs are attached to the body at fixed positions $\mathbf{a}_{i}$ in $\mathcal{B}$. The body has mass $m$ and inertia $I$, with its position and the orientation in $\mathcal{W}$ denoted by $\mathbf{b}$ and $\alpha$, respectively.

Each leg can independently be either in stance or flight, resulting in a hybrid dynamic system model. Legs in stance are assumed stationary at $\mathbf{f}_{i}$ with no slippage. However, toe dynamics during flight are modeled with small masses $m_{t} \ll m$ at each toe, assuming that body dynamics remain unaffected by legs in flight. All legs are composed of a radial spring with stiffness $k_{i}$ and a viscous damper with coefficient $d_{i}$. As in the RHex platform, each hip joint is independently controlled through a torque input $\tau_{i}$. Further details of the equations of motion for this model can be found in [22, 24].

\section{Closed Loop Control of Pronking}

\section{A. Structure of the Flight Controller}

As described in Section II-B, control of SLIP locomotion is achieved by proper selection of control inputs at every apex event. We now associate the apex event for the Slimpod model's flight with the SLIP apex and invoke the corresponding deadbeat controller to find suitable control inputs for the following stride. The flight controller for Slimpod will then try to achieve these control inputs (i.e. touchdown angle and leg precompression) for the virtual leg attached to the body COM by position control of individual leg angles.

1) Leg Control During Flight: The flight controller for pronking must both ensure simultaneous touchdown of all three legs, and also make sure that desired SLIP control inputs can be realized by explicit placement of the virtual toe. To this end, it continuously solves kinematic equations

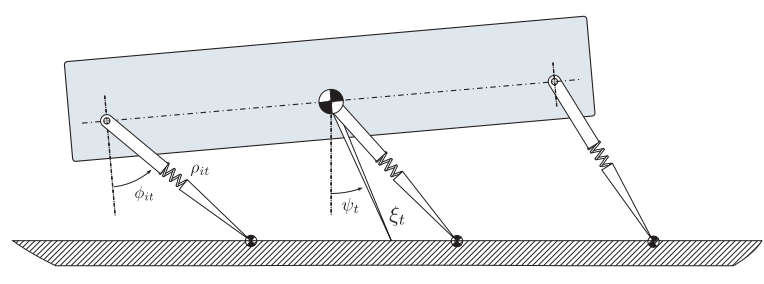

Fig. 4. Leg kinematics at the time of touchdown

for all legs and applies PD control to bring them to desired locations as illustrated in Fig. 4. Based on the SLIP control decisions $\psi_{t d}$ and $\xi_{t d}$, target leg angles are given by

$$
\begin{aligned}
\phi_{i t}^{*} & =\arccos \left(p_{i z} / \rho_{0}\right)-\alpha_{t d} \\
{\left[\begin{array}{c}
p_{i y} \\
p_{i z}
\end{array}\right] } & =\xi_{t d}\left[\begin{array}{c}
\sin \psi_{t d} \\
\cos \psi_{t d}
\end{array}\right]+R\left(\alpha_{t d}\right) \mathbf{a}_{i}
\end{aligned}
$$

where $\phi_{i t}^{*}$ are the target leg angles to generate this pose, realized with feedback control of the hips as

$$
\tau_{i}=-K_{\phi}\left(\phi_{i}-\phi_{i}^{*}\right)-K_{\dot{\phi}} \dot{\phi}_{i} .
$$

Since a numerical estimate of the pitch angle at touchdown, $\alpha_{t d}$ may not be very accurate, our controller simply uses the current, measured pitch angle $\alpha$ in (9), which yields the same result at the moment of touchdown.

2) Virtual Foot Placement: We consider the SLIP template to have transitioned into stance as soon as at least one of the physical legs touches the ground. Following this event, the virtual toe location is determined by the controller, also defining the coordinate frame $\mathcal{V}$ for the following stride. Since actuator limitations of the RHex platform do not admit physical precompression of its legs, our use of a virtual toe to achieve precompression is necessary. After touchdown, embedding controls will attempt to realize template dynamics within the newly formed virtual toe frame.

Due to the nontrivial flight dynamics of each Slimpod leg, the virtual toe placement as anticipated by the flight leg controller may not be exact. In those cases, we select the best possible virtual toe location by giving priority to the touchdown angle over the leg precompression value. Final decisions for the adjusted touchdown angle and the length of the virtual leg hence take the form

$$
\begin{aligned}
\overline{\psi_{t d}} & =\psi_{t d}, \\
\overline{\xi_{t d}} & =b_{z, t d} / \cos \psi_{t d} .
\end{aligned}
$$

where $b_{z, t d}$ is the height of the body COM at touchdown. After the placement of the virtual toe, we introduce polar virtual leg coordinates $\xi$ and $\psi$ in $\mathcal{V}$ and the pitch angle $\alpha$

$$
\mathbf{c}:=[\xi, \psi, \alpha]^{T} \text {. }
$$

\section{B. Control of Stance Dynamics}

At the core of our control methodology is the realization of the template SLIP dynamics for the COM through feedback control during stance. In other words, we would like the COM dynamics in $\mathcal{V}$ to closely match those of the SLIP template. To this end, let $K_{\xi}, K_{\psi}$ and $K_{\alpha}$ be the forcing elements acting on the body COM, induced on the virtual toe coordinates as a result of Slimpod dynamics. We define the 
stance forcing vector resulting from radial leg forces, $\mathbf{F}_{r, s}$, and hip torques, $\tau_{s}$ as

$$
\mathbf{K}:=\left[K_{\xi}, K_{\psi}, K_{\alpha}\right]^{T}=\left(D_{c} \boldsymbol{\phi}\right) \boldsymbol{\tau}_{s}+\left(D_{c} \boldsymbol{\rho}\right) \mathbf{F}_{r, s},
$$

where $D_{c} \boldsymbol{\phi}$ and $D_{c} \boldsymbol{\rho}$ denote the Jacobian matrices of hip angles and leg lengths with respect to the virtual leg coordinates $\xi, \psi$ and $\alpha$. In the following sections, we will define $\mathbf{J}:=D_{c} \boldsymbol{\phi}$ and use $\mathbf{J}_{\psi}$ and $\mathbf{J}_{\psi, \alpha}$ to denote the rows of $\mathbf{J}$ associated with $\psi$ and $\psi, \alpha$, respectively.

Our method forces the stance dynamics of the anchor to closely parallel those of the SLIP template, whose dynamics are characterized by a compliant radial force. Combined with a simple PD pitch stabilizing torque, this requires that

$$
\mathbf{K}=\left[U^{*}(\xi), 0,-C_{\alpha} \alpha-C_{\dot{\alpha}} \dot{\alpha}\right] .
$$

Here, $U^{*}(\xi)$ is the desired radial spring potential law and the null component forces the total moment acting about the virtual toe frame to be zero. In configurations where $\mathbf{J}$ is invertible and all three legs are in stance, leg torques necessary to realize the desired forcing vector are given by

$$
\boldsymbol{\tau}_{s}=\mathbf{J}^{-1}\left(\mathbf{K}^{*}-\mathbf{B}\right)
$$

where $\mathbf{B}:=\left(D_{c} \boldsymbol{\rho}\right) \mathbf{F}_{r, s}$. Unfortunately, $\mathbf{J}$ often ends up rank deficient for most robot configurations during pronking, particularly in the $\xi$ direction [22], making direct inversion impossible. However, the structure of the Jacobian suggests that the radial component is dominated by the robot's passive dynamics, which are already compliant and close to what the SLIP template is trying to achieve. Consequently, we propose an approximate solution and only consider the remaining two virtual toe coordinates, yielding the solution

$$
\boldsymbol{\tau}_{\psi, \alpha}=\mathbf{J}_{\psi, \alpha}^{T}\left(\mathbf{J}_{\psi, \alpha} \mathbf{J}_{\psi, \alpha}^{T}\right)^{-1}\left(\left[0, M_{\alpha}^{*}\right]-\mathbf{B}_{\psi, \alpha}\right)
$$

that minimizes all hip torques while satisfying the angular and pitch dynamics for the COM under the assumption that associated components of the Jacobian are not singular.

In order to ensure practical applicability of our controller, we also impose limits on hip torques based on RHex's actuator specifications. Moreover, we impose additional constraints to prevent premature leg liftoff which often causes instability associated with loss of actuation degrees of freedom. These constraints yield an allowable torque space

$$
\overline{\mathcal{T}}=\left\{\boldsymbol{\tau} \mid \tau_{i, \min } \leq \tau_{i} \leq \tau_{i, \max }\right\} .
$$

In cases where torques returned by (16) are outside this range, we prioritize the angular momentum around the virtual toe, defining the associated feasible torque space as

$$
\mathcal{T}_{\psi}:=\left\{\boldsymbol{\tau} \mid \mathbf{J}_{\psi} \boldsymbol{\tau}+\mathbf{B}_{\psi}=0\right\}
$$

whose elements can be written as $\tau=\tau_{\psi}+\tau_{\perp}$, where

$$
\begin{aligned}
& \boldsymbol{\tau}_{\perp} \in \mathcal{N} \text { ullspace }\left(\mathbf{J}_{\psi}\right) \\
& \boldsymbol{\tau}_{\psi}=\mathbf{J}_{\psi}^{T}\left(\mathbf{J}_{\psi} \mathbf{J}_{\psi}^{T}\right)^{-1} \mathbf{B}_{\psi}
\end{aligned}
$$

In situations where this set of torques intersects the allowable torque space $\overline{\mathcal{T}}$, we find the best choice using the equation

$$
\boldsymbol{\tau}_{s}=\min _{\boldsymbol{\tau} \in\left(\boldsymbol{\tau}_{\psi} \cap \overline{\mathcal{T}}\right)}\left\|\boldsymbol{\tau}-\boldsymbol{\tau}_{\psi, \alpha}\right\|
$$

which is solvable with simple linear programming methods. Otherwise, if $\tau_{\psi} \cap \overline{\mathcal{T}}=\emptyset$, then the best solution is

$$
\boldsymbol{\tau}_{s}=\min _{\boldsymbol{\tau} \in \overline{\mathcal{T}}} \frac{\boldsymbol{\tau}_{\psi}^{T}\left(\boldsymbol{\tau}-\boldsymbol{\tau}_{\psi}\right)}{\left\|\boldsymbol{\tau}_{\psi}\right\|}
$$

Further details of these derivations can be found in [22].

\section{Handling Partial Touchdown and Liftoff}

The embedding algorithm of Section IV-B was formulated under the assumption that all three legs are in stance. However, close to touchdown and liftoff events and particularly in the presence of noise, the number of legs in stance may be smaller. The embedding solution of (14) still applies when only two legs are on the ground but a recovery strategy must be introduced when only a single leg is in stance.

Earlier work on pronking [17] and our preliminary simulations showed that pitch instability is the dominant mode of failure for this behavior. Moreover, control affordance of a single leg is usually much more pronounced in the pitch degree of freedom. Consequently, when only a single leg is in contact with the ground, we only enforce the pitch stabilization goal with $\tau_{s}=J_{\alpha}^{-1}\left(M_{\alpha}^{*}-B_{\alpha}\right)$, limited to the allowed range for the leg motor.

\section{Corrections for Damping and Embedding Inaccuracies}

One of the most significant sources of error in the embedding controller for alternating tripod running in [22] was the presence of damping, making explicit control of running height very difficult. In this section, we introduce a number of model-inspired corrections that enables us to explicitly control the apex height during pronking. Pronking was not possible at all in the absence of these corrections due to the apex height not being properly regulated and legs prematurely touching the ground during protraction.

We first assume that the total damping force acting on the center of mass can be represented as a viscous damping force on the virtual leg, $F_{d}:=d_{s} \dot{\xi}(t)$, where $d_{s}$ is the sum of damping coefficients for all legs. Under this assumption, the total damping losses are given by

$$
\Delta E_{d}=\int_{0}^{T_{s}} d_{s} \dot{\xi}^{2}(t) d t,
$$

where $T_{s}$ is the duration of stance. Assuming that $\dot{\xi}_{t}, \dot{\xi}_{l}$ and $T_{s}$ do not change substantially across strides at steady-state, we fit a sinusoidal function to these data points to obtain an estimate of the radial trajectory $\dot{\xi}(t)$. The energy loss estimated through this approximate trajectory is then added to the energy input $\Delta E$.

The second problem comes from our assumption that passive leg dynamics will automatically yield the desired radial SLIP dynamics with $K_{\xi}=U^{*}(\xi)$. However, due to kinematic differences introduced by the virtual toe placement and nonzero pitch angles, the embedding controller always gains more energy than the target SLIP model. Finding an analytic estimate for this difference is very difficult due to 


\begin{tabular}{|c|c|c|}
\hline \multicolumn{1}{|c|}{ Hip attachment coordinates of the legs } \\
\hline$a_{1}$ & $a_{2}$ & $a_{3}$ \\
\hline$[-0.24 ; 0]$ & {$[0 ; 0]$} & {$[0 ;-0.24]$} \\
\hline \hline \multicolumn{3}{|c|}{ Inertial parameters } \\
\hline$m$ & $m_{t}$ & $I$ \\
\hline $9 k g$ & $0.1 \mathrm{~kg}$ & $0.2 \mathrm{~kg} \mathrm{~m} \mathrm{~m}^{2}$ \\
\hline \multicolumn{3}{|c|}{ Leg parameters } \\
\hline$\rho_{0}$ & $\mathrm{k}$ & $\mathrm{d}$ \\
\hline $0.19 \mathrm{~m}$ & $4000 \mathrm{~N} / \mathrm{m}$ & $24 \mathrm{Nm} / \mathrm{s}$ \\
\hline \hline \multicolumn{3}{|c|}{ Motor Characteristics } \\
\hline Stall Torque & Max Speed & Torque Loss \\
\hline $36.7 \mathrm{~N} \mathrm{~m}$ & $49.4 \mathrm{rad} / \mathrm{s}$ & 0.1 \\
\hline
\end{tabular}

TABLE II

Kinematic and Dynamic Parameters of the Slimpod Model
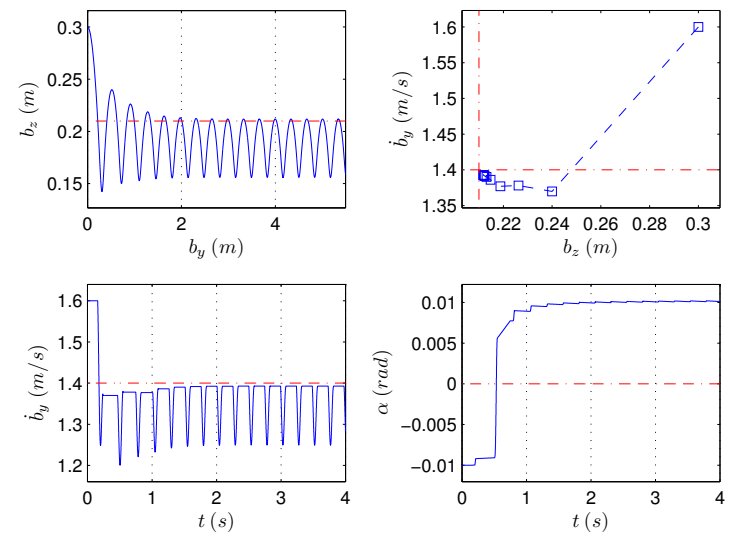

Fig. 5. An example simulation of duration $7 s$, from an initial condition with $b_{z}=0.3 \mathrm{~m}, b_{\dot{y}}=1.6 \mathrm{~m} / \mathrm{s}, \alpha=-0.01 \mathrm{rad}$ and the apex goal selected as $b_{z}^{*}=0.21 \mathrm{~m}, b_{\dot{y}}^{*}=1.4 \mathrm{~m} / \mathrm{s}$.

the complex morphology of the system. Consequently, we modify the selection of the leg precompression in (1) as

$$
\xi_{t d}=\xi_{0}-\sqrt{2\left(K_{E} \Delta E\right) / k},
$$

where $K_{E}$ is an energy scaling factor, experimentally tuned to be 0.11 for our simulations. Finally, we impose an upper limit on the amount of precompression which causes legs to remain much closer to parallel during stance and significantly improves the energetic accuracy of the embedding controller.

\section{RESUlTS AND DiscUSSION}

This section presents our simulation results, analyzing the existence and stability of limit cycles as well as the tracking accuracy of the proposed controller both for an ideal simulation and under state measurement noise. All experiments were run in Matlab, within a simulation toolbox whose results we verified against SimSect [21].

Table II details kinematic and dynamic parameters of the Slimpod model used throughout our simulations. These parameters were selected to closely match the morphology and properties of our physical hexapod RHex to support practical applicability of the proposed method.

\section{A. Existence and Nature of Stable Limit Cycles}

Fig. 5 illustrates an example pronking run with no noise, starting from an arbitrary initial condition and converging to the selected goal of $b_{z}^{*}=0.21 \mathrm{~m}, b_{\dot{y}}^{*}=1.4 \mathrm{~m} / \mathrm{s}$. Locomotion

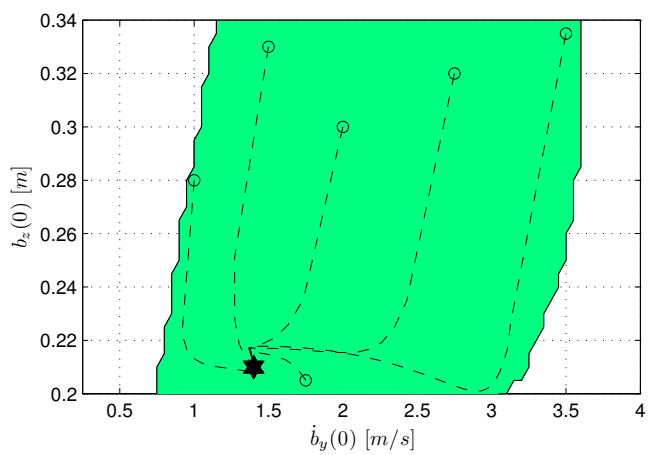

Fig. 6. Cross section $\left(\dot{b}_{y}-b_{z}\right)$ of the stable domain of attraction for the goal $\dot{b}_{y}^{*}=1.4 \mathrm{~m} / \mathrm{s}$ and $b_{z}^{*}=0.21 \mathrm{~m}$. Green region illustrates initial conditions from which the hexapod converges to stable pronking in $7 \mathrm{~s}$. Dashed lines illustrate a few example runs to show convergence behavior.

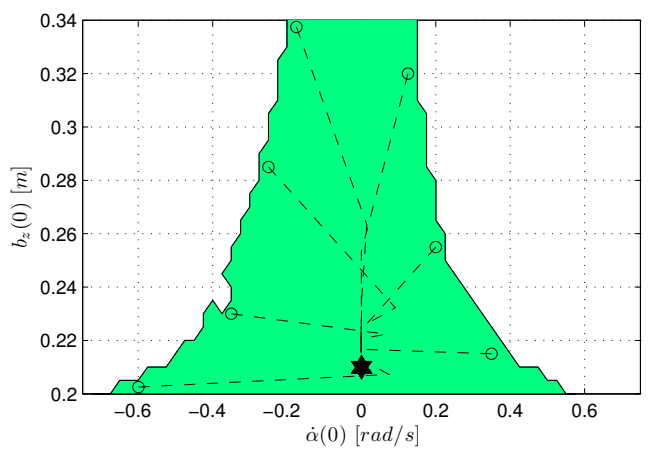

Fig. 7. Cross section $\left(\dot{\alpha}-b_{z}\right)$ of the stable domain of attraction for the goal $\dot{b}_{y}^{*}=1.4 \mathrm{~m} / \mathrm{s}$ and $b_{z}^{*}=0.21 \mathrm{~m}$. Green region illustrates initial conditions from which the hexapod converges to stable pronking in $7 \mathrm{~s}$. Dashed lines illustrate a few example runs to show convergence behavior.

quickly converges to a limit cycle with very small steadystate errors indicating that both the embedding controller and the SLIP deadbeat controller are performing well. In all of our simulations, we observed that the controller either converges to a single, stable limit cycle, or irrecoverably fails due to a structural faults such as toe stubbing or the robot body colliding with the ground. No controller parameters or initial conditions produced period-two or more oscillations.

\section{B. Stability and Basins of Attraction}

In order to generalize the results shown in Fig. 5 and more accurately characterize the stability properties of the pronking controller, we systematically ran simulations from a variety of different initial conditions toward the same goal setting of $b_{z}^{*}=0.21 \mathrm{~m}, b_{\dot{y}}^{*}=1.4 \mathrm{~m} / \mathrm{s}$. We considered a run stable if the apex states of the last 5 steps were within $1 \%$ of their average. Two different cross-sections of the resulting region of attraction are shown in Figs. 6 and 7. Even though it is not surprising to see that pronking fails at very high speeds (above $3.2 \mathrm{~m} / \mathrm{s}$ ), it also does not perform well for slow speeds. We believe that this is primarily due to the underactuated nature of our platform which becomes unable to inject energy into the system at slow speeds where leg angles are shallow and the effects of leg torques are primarily 


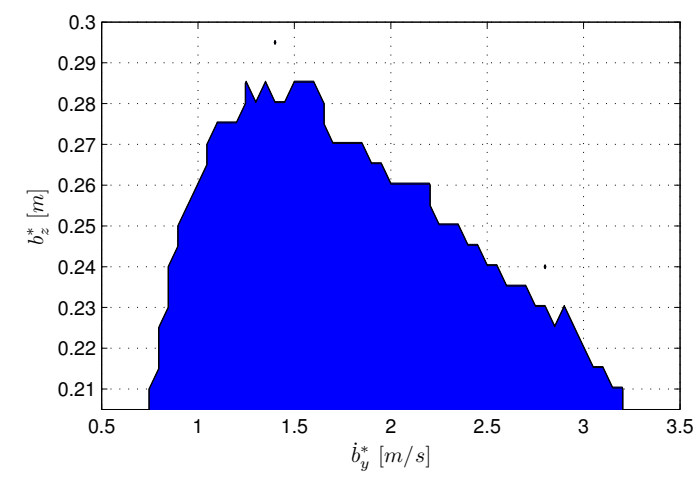

Fig. 8. Maneuverability of the pronking controller. The blue region illustrates the set of apex goal settings for which stable pronking is possible.

in the forward direction. Nevertheless, this does not present a serious problem since the pronking behavior is considered useful mainly for achieving medium to high speeds.

\section{Maneuverability}

As noted before, one of the primary contributions of this paper is the maneuverability of pronking. To show this, we ran a series of simulations with different apex goal settings, starting from initial conditions close to the goal. As in the previous section, we identified goal settings for which stable pronking was possible by checking the last 5 apex states and making sure they are within $1 \%$ of their average and within $5 \%$ of the desired goal.

Our results in Fig. 8 show that pronking speed and height can be explicitly controlled within a very large region using our embedding controller. There seems to be a preferred speed of approximately $1.5 \mathrm{~m} / \mathrm{s}$ at which the largest apex height can be controllably achieved. However, given the leg length of $0.19 \mathrm{~m}$ for our RHex platform, stable pronking at apex heights as large as $0.26 \mathrm{~m}$ are achievable, corresponding to previously unachieved flight phases for this robot.

\section{Noise Performance}

Our final set of simulations investigate controller performance under substantial noise conditions. Firstlt, while the results of the previous section were obtained through continuous integration of controller equations with system dynamics, we now discretize our controller actions and apply torque commands at a frequency of $1 \mathrm{KHz}$ with zero-order hold. Results under this update frequency are much more realistic since any physical robotic platform will have the a similar constraint while performing closed loop control.

In addition to this "discretization noise", we also add zero-mean, white gaussian noise of variance $2 \%$ to our force, velocity and pitch rate measurements in an attempt to realistically model sensory noise that would be present in a physical robot. Noise in positional measurements is less critical since instrumented legs often provide rather accurate estimates of both leg configurations as well as body position during stance. Fig. 9 illustrates the same example run as Fig. 5 under these noise conditions. This example shows that
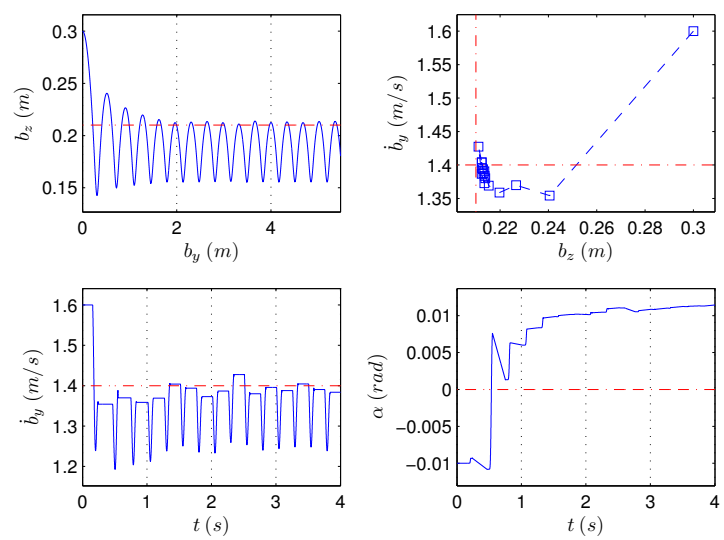

Fig. 9. An example noisy discrete simulation from an initial condition with $b_{z}=0.3 \mathrm{~m}, b_{\dot{y}}=1.6 \mathrm{~m} / \mathrm{s}, \alpha=0.01 \mathrm{rad} / \mathrm{s}$ and the apex goal selected as $b_{z}^{*}=0.21 \mathrm{~m}, b_{\dot{i}}^{*}=1.4 \mathrm{~m} / \mathrm{s}$.

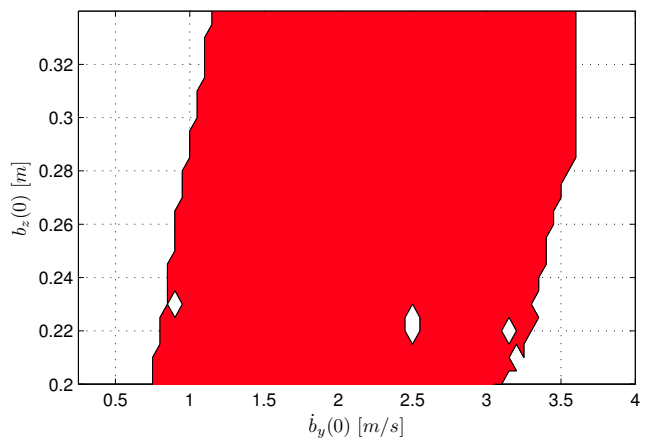

Fig. 10. Cross section $\left(\dot{b}_{y}-b_{z}\right)$ of the stable domain of attraction for the goal $\dot{b}_{y}^{*}=1.4 \mathrm{~m} / \mathrm{s}$ and $b_{z}^{*}=0.21 \mathrm{~m}$. Red region illustrates initial conditions from which the hexapod converges to stable pronking in $7 \mathrm{~s}$.

the added noise leads to degraded steady-state performance in pronking, but the nature of the limit cycle remains the same. simulations to identify the domain of attraction for the pronking behavior under the aforementioned noise conditions. Red regions in Figures 10 and 11 show two cross sections of this region of attraction, which are non suprisingly slightly smaller than their counterparts under no noise conditions, but are still substantial for the pronking behavior. These results show that the embedding controller is robust against the kinds of noise that would be typically observed for a physical platform.

Clearly, there are still numerous challenges toward the deployment of this behavior on a physical robot. However, the robustness of our controller to at least the most basic types of discretization and state measurement noise suggest that such a deployment is not entirely infeasible.

\section{CONCLUSIONS AND Future WORK}

In this paper, we introduced a novel feedback controller to achieve stable and maneuverable control of hexapedal pronking. Our method is based on active embedding of simple template dynamics, the SLIP model in our case, into a more complex hexapedal morphology. The end result is a clean separation of a simple dynamical model for the spec- 


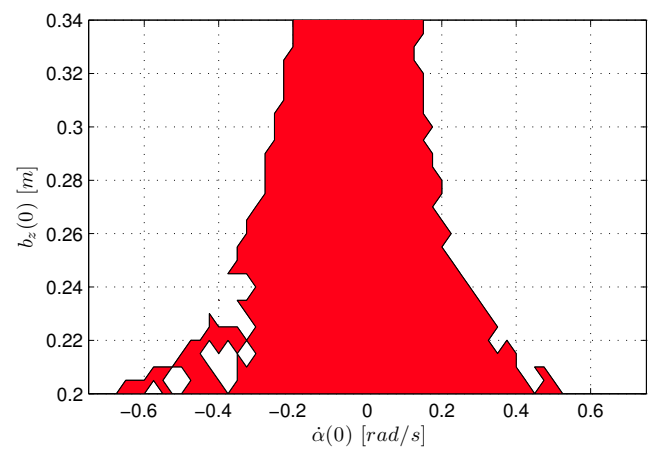

Fig. 11. Cross section $\left(\dot{\alpha}-b_{z}\right)$ of the stable domain of attraction for the goal $\dot{b}_{y}^{*}=1.4 \mathrm{~m} / \mathrm{s}$ and $b_{z}^{*}=0.21 \mathrm{~m}$. Red region illustrates initial conditions from which the hexapod converges to stable pronking in $7 \mathrm{~s}$.

ification and control of higher level task parameters, while remaining degrees of freedom are independently controlled and stabilized.

The novelty of our work is both in achieving successful pronking in the presence of severe underactuation and in careful characterization of the performance of the resulting control algorithm under noise due to both discrete control and measurement uncertainty. We provided simulation evidence to establish the existence and stability of limit cycles with large basins of attraction. We also established that the resulting controller nicely illustrates the maneuverability advantages presented by the template abstraction, with a large region of possible locomotion speeds and heights across which explicit control is possible. Both the stability regions and maneuverability properties of our controller were found to be superior to those that were obtained for alternating tripod gaits in [24]. We believe that the realization of this algorithm on the experimental RHex platform will be possible based on our results with realistic disturbances in the form of discretization and measurement noise.

In the long term, we would like to reduce the dependence of the pronking controller on high bandwidth state measurements, resulting in more open-loop controllers through careful investigation and analysis of the results obtained from closed-loop pronking. This will eventually enable the RHex platform to add pronking to its repertoire of robust behaviors that it can safely deploy in the outdoors. Finally, we also believe that pronking is the most likely behavior on which the template based control strategy, so far only successfully applied in simulation, will be physically realized.

\section{ACKNOWLEDGEMENTS}

M. Mert Ankaralı was partially supported by TUBITAK, the Scientific and Technical Research Council of Turkey.

\section{REFERENCES}

[1] R. M. Alexander and A. S. Jayes. Vertical movement in walking and running. Journal of Zoology, London, 185:27-40, 1978.

[2] T. Allen, R. Quinn, R. Bachmann, and R. Ritzmann. Abstracted biological principles applied with reduced actuation improve mobility of legged vehicles. In Intelligent Robots and Systems, 2003. (IROS 2003). Proceedings. 2003 IEEE/RSJ International Conference on, volume 2, pages 1370-1375 vol.2, Oct. 2003.
[3] R. e. Altendorfer. Evidence for spring loaded inverted pendulum running in a hexapod robot. In D. Rus and S. Singh, editors, Experimental Robotics VII, Lecture Notes in Control and Information Sciences, chapter 5, pages 291-302. Springer, December 2000.

[4] M. Berkemeier and P. Sukthankar. Self-organizing running in a quadruped robot model. In Proceedings of the International Conference on Robotics and Automation, pages 4108-4113, April 2005.

[5] M. D. Berkemeier. Modeling the Dynamics of Quadrupedal Running. The International Journal of Robotics Research, 17(9):971-985, 1998.

[6] R. Blickhan. The spring-mass model for running and hopping. Journal of Biomechanics, 22:1217-1227, 1989.

[7] R. Blickhan and R. J. Full. Similarity in multilegged locomotion: Bouncing like a monopode. Journal of Comparative Physiology A: Neuroethology, Sensory, Neural, and Behavioral Physiology, 173(5):509-517, Nov. 1993.

[8] T. M. Caro. Ungulate antipredator behaviour: Preliminary and comparative data from african bovids. Behavior, 128(3-4):189-228, 1994.

[9] P. Chatzakos and E. Papadopoulos. Bio-inspired design of electricallydriven bounding quadrupeds via parametric analysis. Mechanism and Machine Theory, 44(3):559 - 579, 2009. Special Issue on Bio-Inspired Mechanism Engineering.

[10] C. Chevallereau, E. R. Westervelt, and J. W. Grizzle. Asymptotically Stable Running for a Five-Link, Four-Actuator, Planar B ipedal Robot. The International Journal of Robotics Research, 24(6):431-464, 2005.

[11] C. T. Farley, J. Glasheen, and T. A. McMahon. Running springs: Speed and animal size. Journal of Experimental Biology, 185:71-86, 1993.

[12] C. D. FitzGibbon and J. H. Fanshawe. Stotting in thomson's gazelles: an honest signal of condition. Behavioral Ecology and Sociobiology, 23(2):69-74, Aug. 1988.

[13] R. J. Full and D. E. Koditschek. Templates and anchors: Neuromechanical hypotheses of legged locomotion. Journal of Experimental Biology, 202:3325-3332, 1999.

[14] H. Geyer, A. Seyfarth, and R. Blickhan. Spring-mass running: simple approximate solution and application to gait stability. Journal of Theoretical Biology, 232(3):315-328, Feb. 2005.

[15] P. Gregorio, M. Ahmadi, and M. Buehler. Design, control, and energetics of an electrically actuated legged robot. Transactions on Systems, Man, and Cybernetics, 27(4):626-634, August 1997.

[16] J. W. Grizzle, G. Abba, and F. Plestan. Asymptotically stable walking for biped robots: analysis via systems with impulse effects. IEEE Transactions on Automatic Control, 46(1):51-64, January 2001.

[17] D. McMordie and M. Buehler. Towards pronking with a hexapod robot. In 4th Int. Conf. on Climbing and Walking Robots, Karlsruhe, Germany, 2001.

[18] G. M. Nelson and R. D. Quinn. Posture control of a cockroach-like robot. IEEE Control Systems Magazine, 19(2):9-14, 1999.

[19] I. Poulakakis and J. W. Grizzle. Formal embedding of the spring loaded inverted pendulum in an asymmetric hopper. In Proceedings of the European Control Conference, 2007.

[20] M. Raibert. Legged robots that balance. MIT Press series in artificial intelligence. MIT Press, Boston, 1986.

[21] U. Saranli. SimSect hybrid dynamical simulation environment. Technical Report CSE-TR-436-00, UM, Ann Arbor, MI, 2000.

[22] U. Saranli. Dynamic Locomotion with a Hexapod Robot. PhD thesis, The University of Michigan, Ann Arbor, MI, September 2002.

[23] U. Saranli, M. Buehler, and D. E. Koditschek. RHex: A simple and highly mobile robot. International Journal of Robotics Research, 20(7):616-631, July 2001.

[24] U. Saranli and D. E. Koditschek. Template based control of hexapedal running. In Proc. of the IEEE Int. Conf. On Robotics and Automation, volume 1, pages 1374-1379, Taipei, Taiwan, September 2003.

[25] U. Saranli, W. J. Schwind, and D. E. Koditschek. Toward the control of a multi-jointed, monoped runner. In Proceedings of the IEEE International Conference On Robotics and Automation, volume 3, pages 2676-82, New York, 1998.

[26] A. Sato and M. Buehler. A planar hopping robot with one actuator: design, simulation, and experimental results. In Proceedings of the International Conference on Intelligent Robots and Systems, volume 4, pages 3540-3545, Sept.-2 Oct. 2004.

[27] W. J. Schwind. Spring Loaded Inverted Pendulum Running: A Plant Model. Phd, University of Michigan, 1998.

[28] G. Z. Zeiglin and H. B. Brown. Control of a bow leg hopping robot. In In Proceedings of the IEEE International Conference On Robotics and Automation, volume 1, pages 793-798, May 1998. 